\title{
MCT APD DETECTION SYSTEM FOR ATMOSPHERIC PROFILING APPLICATIONS USING TWO-MICRON LIDAR
}

\author{
Tamer F. Refaat", Mulugeta Petros, Ruben Remus and Upendra N. Singh \\ NASA Langley Research Center, Hampton, Virginia 23681, USA \\ *Email: tamer.f.refaat@nasa.gov
}

\begin{abstract}
An advanced detection system, based on mercury cadmium telluride avalanche photodiodes array, was implemented within a $2-\mu \mathrm{m}$ lidar. Detection system characterization was conducted for performance evaluation including settling time, noise-equivalent-power (NEP) and dynamic range. Results indicated an average NEP of $1.4 \mathrm{fW} / \mathrm{Hz}^{1 / 2}$ per pixel. Lidar range resolved profiling demonstrated the dynamic range capability by measuring near-field aerosol scattering and farfield clouds reflection simultaneously.
\end{abstract}

\section{INTRODUCTION}

An airborne triple-pulse integrated path differential absorption (IPDA) lidar has been developed at NASA Langley Research Center [13]. This instrument measures both atmospheric carbon dioxide $\left(\mathrm{CO}_{2}\right)$ and water vapor $\left(\mathrm{H}_{2} \mathrm{O}\right)$ simultaneously. The IPDA transmitter produces high-energy laser pulses at 2- $\mu \mathrm{m}$ wavelength with a repetition rate of $50 \mathrm{~Hz}$. Each laser shot consists of a burst of three $50 \mathrm{~ns}$ pulses, separated by 200 $\mu$ s, with a different wavelength setting for each pulse [4]. The operating wavelengths of the three pulses were selected, with respect to the $\mathrm{CO}_{2} \mathrm{R} 30$ line center, to target $\mathrm{H}_{2} \mathrm{O}$ absorption, $\mathrm{CO}_{2}$ absorption and minimum absorption (off-line), for the first, second and third pulses, respectively [1]. The IPDA receiver consists of a $0.4 \mathrm{~m}$ Newtonian telescope that focuses the return radiation onto a $300 \mu \mathrm{m}$ spot size. The return radiation is collimated and filtered, before being split (90\%$10 \%)$ into high and low signal channels. The high signal channel is focused onto a $300-\mu \mathrm{m}$ diameter commercial extended-range InGaAs PIN photodetector. The low signal channel is used to extend the detection dynamic range for high returns without saturation. In addition, the low signal channel can be used to test other $2-\mu \mathrm{m}$ detection technologies [3].

Recent developments of advanced single-chargecarrier Mercury Cadmium Telluride ( $\mathrm{HgCdTe}$ or
MCT) electron-initiated avalanche photodiodes (e-APD) are a breakthrough in lidar detection technology [5-6]. Compared to other detector materials, MCT is a mature and reliable technology amenable to space qualification. Several IPDA lidar validations were conducted using MCT e-APD detection systems. These validations were focused on the $1.6 \mu \mathrm{m}$ wavelength targeting $\mathrm{CO}_{2}$, methane and oxygen using the IPDA lidar technique [7-9]. Recently, an MCT e-APD detection system was integrated and characterized for the 2- $\mu \mathrm{m}$ wavelength [10]. In this paper, atmospheric testing of the detection system using the $2-\mu \mathrm{m}$ triple-pulse IPDA lidar is presented. Focusing on the off-line return, the MCT e-APD direct detection system exhibited a dynamic range and sensitivity suitable for atmospheric profiling within the boundary layer, while observing far-field clouds.

\section{MCT E-APD DETECTION SYSTEM}

The MCT e-APD consists of 16 pixels arranged in $4 \times 4$ pixel-array with an $80 \times 80 \mu^{2}$ pixel-size. This device operates at $77 \mathrm{~K}$ and exhibits IR sensitivity from $1-\mu \mathrm{m}$ up to $4-\mu \mathrm{m}$ [5]. The e-APD is connected to a readout integrated circuit (ROIC) that accesses each pixel through individual, variable gain, trans-impedance amplifiers (TIA). An output summing amplifier (SUM) produces the summation of a specific number of pixels. Pixel activation is selected by software, which controls device operation and allows changing the bias voltage and the TIA gains. A fixed gain buffer amplifier, for driving $50 \Omega$ loads, follows the SUM. The TIA and SUM are coupled through either $\mathrm{dc}$ or ac coupling using hardware connectors. The detection system output is limited to $1.4 \mathrm{~V}[6,10]$.

The 2- $\mu \mathrm{m}$ e-APD detection system includes a vacuum system, which allows cooling the device using a cryo-cooler to reduce dark current and noise. A cold narrow band-pass optical filter was integrated inside the chamber to transmit $2-\mu \mathrm{m}$ 
and reduce background radiation. Additional custom designed aft-optics couples the input radiation to an optical fiber, and focuses it onto the center $2 \times 2$ pixels. Limiting the number of active pixels reduces the noise of the detection system. The whole system is integrated in a rack mountable chassis at NASA Goddard Space Flight Center [6]. The detection system bandwidth is set to $6 \mathrm{MHz}$. An advanced, 12-bit, $1 \mathrm{GS} / \mathrm{s}$ digitizer is connected to the output for data recording.

\subsection{Impulse Response and Coupling}

Generally, backscatter lidar detection systems are required to acquire both high and low signals. High frequency, high amplitude signals, in the form of pulses, are generated from near-field scattering and hard targets such as ground and cloud returns. Low frequency, low amplitude signals are generated from aerosol scattering, which is characterized by a slowly varying spatial distribution, close to the background signal. Therefore, dc coupling is typically used for backscatter lidar applications. It is important for the detection system to recover quickly from high frequency pulses to avoid bias introduction to the background signal [11]. Thus, pulse short time recovery is an important aspect of the impulse response of the detection system. IPDA is a special lidar technique only focused on hard target returns. In this case, ac coupling can be implemented for IPDA only if the transmitted pulse-width is short compared to the resistancecapacitance (RC) time constant of the coupling.

An experimental setup was used to measure the impulse response of the detection system using different pulse-widths and couplings. A radiation pulse was simulated using a $2-\mu \mathrm{m}$ continuouswave laser and an acousto-optic modulator. The modulator is driven by a pulse generator in order to turn on and off the radiation toward the detector. The pulse-width was varied using a pulse generator. Figure 1 shows the impulse response results of the detection system output at different pulse widths using ac and dc couplings. For longer pulse-width $(100 \mu \mathrm{s})$ ac coupling results in pulse deformation, compared to dc coupling, with long recovery time. The recovery time is measured as the difference between the pulse peak time and the time required to achieve a signal level equal to the pulse pre-trigger. For $1 \mu \mathrm{s}$ pulses recovery times are 1.7 and $0.9 \mu$ s for dc and ac coupling, respectively. These reduce to 1.0 and $0.6 \mu$ s for the $10 \mathrm{~ns}$ pulse. Thus, ac coupling may be favorable for IPDA applications, which results in shorter recovery time for short pulses. In addition, ac coupling reduces low-frequency noise (e.g., $1 / \mathrm{f}$ noise) and preserve detection dynamic range by eliminating dc offsets. This results in an enhanced signal-to-noise ratio (SNR). Besides, ac coupling may underestimate pulse amplitude, as indicated in figure 1, causing IPDA biases.

\subsection{Dynamic Range}

For a fixed bias voltage and gain setting, the detection system dynamic range is defined by the minimum and maximum detectable optical powers in Watt. Minimum detectable power corresponds to the system noise-equivalent-power (NEP in $\mathrm{W} / \mathrm{Hz}^{1 / 2}$ ) multiplied by the square root of the bandwidth. From the NEP definition, this power results at unity SNR. Maximum detectable power is equal to the highest radiation intensity that can be applied to the detection system without causing saturation or clipping in either e-APD detector, electronics or digitizer. This corresponds to the digitizer maximum output voltage divided by gains and responsivity [10].

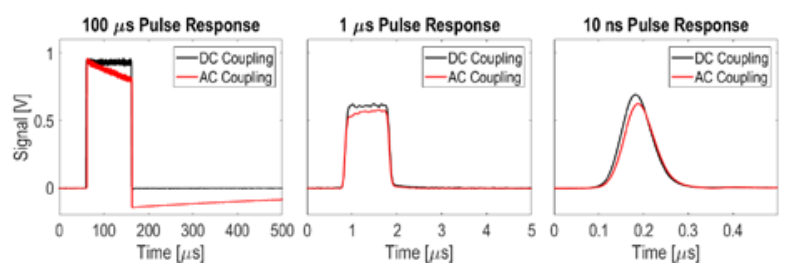

Figure 1. MCT e-APD 2-um detection system impulse response using $d c$ and ac coupling at different radiation pulse-width. Results obtained using gain setting of $0,77.6 \mathrm{~K}$ and $12 \mathrm{~V}$ bias.

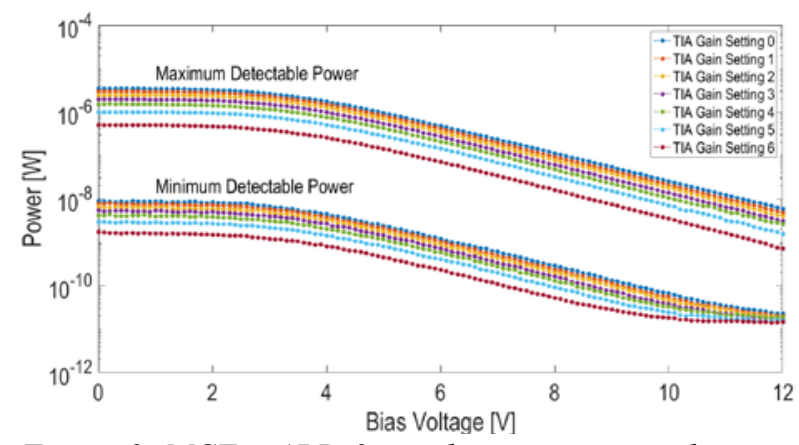

Figure 2. MCT e-APD 2- 4 m detection system dynamic range versus bias voltage using different gains. Results are obtained for the $2 \times 2$ center pixels at $77.6 \mathrm{~K}$. 
These procedures were repeated for different bias voltages and TIA gain settings. Figure 2 shows the dynamic range of the MCT e-APD detection system. These results indicate more than two orders of magnitude input dynamic range, which can be controlled by either the bias or the TIA gain. The absolute minimum detectable power, observed as lower saturation at high bias, is 14 $\mathrm{pW}$, which is independent of TIA gain. This results in a total detection system NEP of 5.7 $\mathrm{fW} / \mathrm{Hz}^{1 / 2}$ for four pixels. This translates to about $1.4 \mathrm{fW} / \mathrm{Hz}^{1 / 2}$ average NEP per pixel.

\section{ATMOSPHERIC PROFILING}

The 2- $\mu \mathrm{m}$ triple-pulse IPDA lidar is installed inside a mobile trailer for ground testing [12]. The instrument is nadir pointing similar to an airborne setup. Two, $0.6 \mathrm{~m}$ diameter, $45^{\circ}$ mirrors are used to convert the IPDA pointing to zenith through a rooftop IR coated window. The objective of this test was to use clouds as hard target to assess the capability of the new MCT e-APD detection system. In these experiments, the detection system was dc coupled and the APD was cooled to $77 \mathrm{k}$.

\subsection{Near-Field Cumulus Clouds}

On December 12, 2017, the IPDA lidar was operated to target near-field cumulus clouds at around $2 \mathrm{~km}$ altitude. Figure 3 shows a sample profile of the off-line return, seeded at 2051.1915 $\mathrm{nm}$, and the range-corrected signal. The MCT eAPD was biased using $7 \mathrm{~V}$, with six active pixels and a TIA gain setting of four. Signal processing included pulse time alignment with respect to the initial window reflection. This is followed by 100 shot averaging $(2 \mathrm{~s})$, background subtraction, and range correction. Figure 4 shows a false color diagram for successive signals acquired during a 30 minute record of the cloud structure.

\subsection{Far-Field Cirrus Clouds}

On December 14, 2017, the IPDA was focused toward high-altitude $(6 \mathrm{~km})$ cirrus clouds. The objective was to test the instrument measurement capability using far-field targets. In this record, four pixels of the MCT e-APD were activated. The device was biased with $8 \mathrm{~V}$ and a TIA gain setting of five. Signal processing included pulse alignment, 50 shot averaging $\left(\begin{array}{ll}1 & \mathrm{sec}\end{array}\right)$ and background subtraction followed by range correction. Figure 5 shows a sample profile for an off-line return signal with the cloud return pulse occurring at $40 \mu \mathrm{s}$. This is equivalent to $6 \mathrm{~km}$ altitude as shown in the range corrected signal in the same figure. Focusing on the near-field range corrected signal reveals aerosol scattering from the boundary layer, as shown in the same figure.
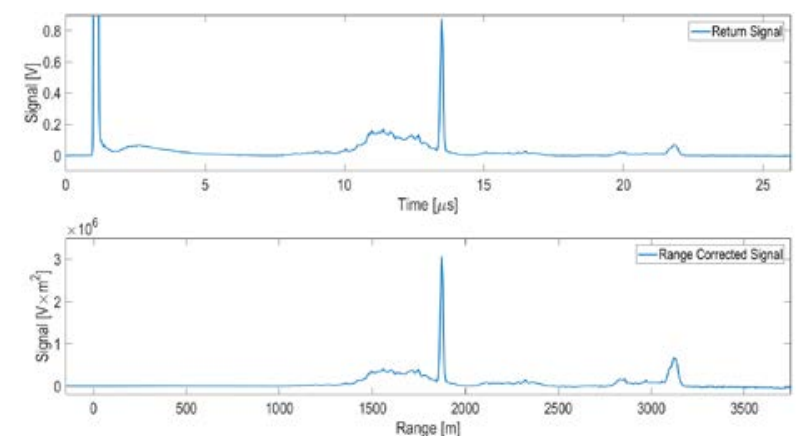

Figure 3. Sample profile of a near-filed cumulus cloud return at 2- $\mu \mathrm{m}$ using MCT e-APD detection system (top) and the range corrected signal (bottom).

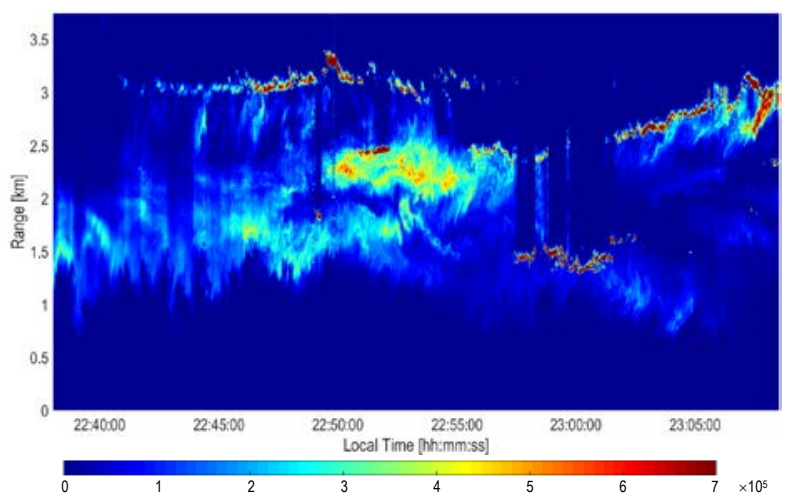

Figure 4. False color diagram for near-field record of cumulus clouds structure. Colors represent the 2- $\mu m$ rang corrected signal strength.

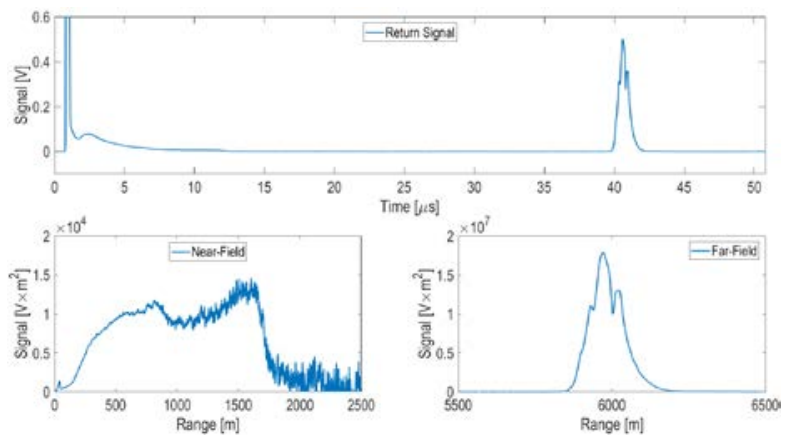

Figure 5. Sample profile of a far-filed cirrus cloud return at 2- $\mu$ m using MCT e-APD detection system (top) and the zoom-in range corrected signal for the nearfield boundary layer (bottom left) and far-filed cloud structure (bottom right). 


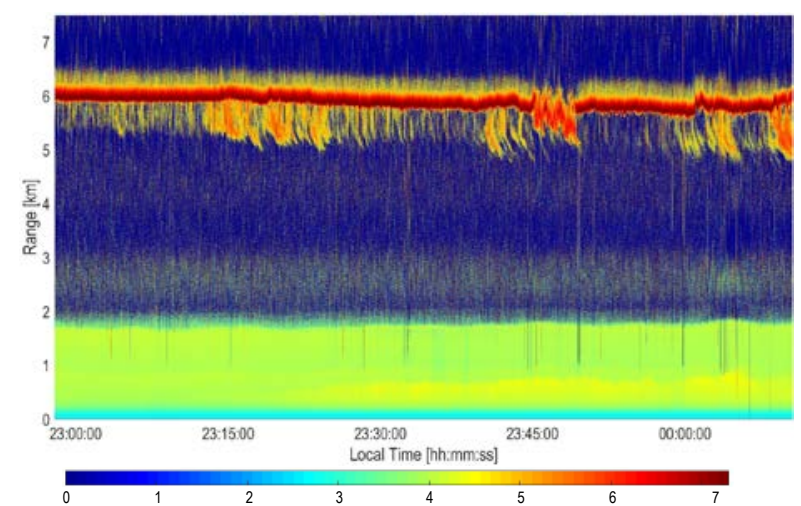

Figure 5. False color diagram for far-field record of cirrus clouds and boundary layer structures. Colors

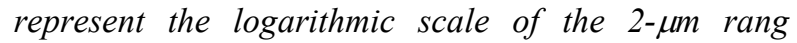
corrected signal strength.

Figure 6 shows a false color diagram for successive signals acquired during a 30 minute recording of cirrus clouds features (including precipitating cirrus) and boundary layer structure. The color scale represents the strength of the range corrected backscatter signal, presented in logarithmic scale, to capture the large dynamic range. Figure 7 illustrates the boundary layer including the altitude distribution of different airmasses. In this figure, false color represents a linear scale of the range corrected signals. MCT eAPD sensitivity and detection system dynamic range enable observing near-field boundary layer structure and far-field clouds simultaneously at the $2-\mu \mathrm{m}$ wavelength. These results, demonstrating both large dynamic range and high sensitivity, enable the potential for space-based $\mathrm{CO}_{2}$ and $\mathrm{H}_{2} \mathrm{O}$ atmospheric profiling using the range-resolved differential absorption lidar (DIAL) technique.

\section{CONCLUSIONS}

NASA Langley Research Center has been developing 2- $\mu \mathrm{m}$ lidar transmitter technologies for Earth science applications. In coordination with NASA's Earth Science Technology Office, NASA Goddard Space Flight Center integrated a 2- $\mu \mathrm{m}$ MCT e-APD lidar detection system. Similar detection systems demonstrated breakthroughs in lidar applications, with maturity amenable to space applications. Combining $2-\mu \mathrm{m}$ transmitter and MCT e-APD detection technologies in a single lidar results in an instrument that could meet the necessary requirements for space-based $\mathrm{CO}_{2}$ measurements.

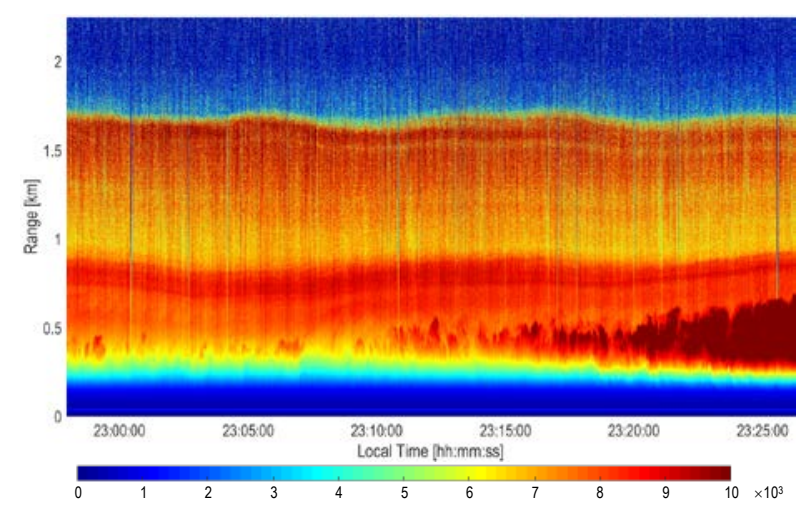

Figure 6. False color diagram for zoom-in near-field boundary layer structure at 2- $\mu$ m. Colors represent range corrected signal strength.

Detection system characterization included the impulse response and dynamic range. $\mathrm{AC}$ coupling enhances SNR, while limited by the RC time constant. AC coupling can underestimate signals amplitudes, which may introduce biases for IPDA applications. Results indicated an average NEP of about $1.4 \mathrm{fW} / \mathrm{Hz}^{1 / 2}$ per pixel. IPDA zenith operation enables assessment of the MCT e-APD sensitivity and detection system dynamic range. The system was capable of observing near-field boundary layer structure and far-field clouds simultaneously at the $2-\mu \mathrm{m}$ wavelength. These results are encouraging for $\mathrm{CO}_{2}$ DIAL profiling applications.

\section{ACKNOWLEDGEMENTS}

This work was funded and supported by NASA's Earth Science Technology Office. The authors acknowledge NASA Goddard Space Flight Center support. Thanks go to Dr. S. Ismail for his input.

\section{REFERENCES}

[1] T. Refaat, et al. Appl. Opt., 54, 1387-1398 (2015).

[2] U. Singh, et al. Proc. of SPIE, 10406-03 (2017).

[3] M. Petros, et al. ILRC 28, 176, 01009 (2018).

[4] U. Singh, et al., Optical Materials Express, 5(4), 824-837 (2015).

[5] J. Beck, et al., J. Elec. Mat., 43, 2970-2977 (2014).

[6] X. Sun, et al. Proc. SPIE, 9114-0K (2014)

[7] J. Abshire, et al., Atmos. Meas. Tech., 11, 20012025 (2018).

[8] H. Riris, et al., J. Appl. Rem. Sen., 11, 034001 (2017).

[9] H. Riris, et al., Opt. Exp., 25, 29307-29327 (2017).

[10] T. Refaat, et al., IEEE IGARSS, 1865-1868 (2018)

[11] A. Dumas, et al., Appl. Opt., 56, 7577-7587 (2017).

[12] T. Refaat, et al. Proc. of SPIE, 10779-02 (2018). 